\title{
The Reaction Entrance Channel and Isotopic Composition of Fragments in a Dynamical and Statistical Multifragmentation Regime
}

\author{
P. M. Milazzo, G. V. Margagliotti, R. Rui, M. Sisto, \\ Dipartimento di Fisica and INFN Trieste, Italy \\ G. Vannini, M. Bruno, M. D’Agostino, \\ Dipartimento di Fisica and INFN Bologna, Italy
}

C. Agodi, R. Alba, G. Bellia, R. Coniglione, A. Del Zoppo, P. Finocchiaro, C. Maiolino, E. Migneco, P. Piattelli, D. Santonocito, P. Sapienza, INFN Laboratori Nazionali del Sud, Catania, Italy

\author{
N. Colonna, \\ INFN Bari, Italy \\ F. Gramegna, P.F. Mastinu, \\ INFN Laboratori Nazionali di Legnaro, Italy \\ I. Iori, and A. Moroni \\ Dipartimento di Fisica and INFN Milano, Italy
}

Received on 13 October, 2003

\begin{abstract}
From the study of the $\mathrm{Ni}+\mathrm{Ni}, \mathrm{Ag} 30 \mathrm{MeV} /$ nucleon dissipative midperipheral collisions, it has been possible to detect events in which Intermediate Mass Fragments (IMF) are emitted by two different sources with different mechanisms. The sources are: a) a quasi-projectile (QP) (and a quasi-target (QT)), with an excitation energy that leads to multifragmentation totally described in terms of a statistical disassembly of a thermalized system (T $\simeq 4 . \mathrm{MeV}, \mathrm{E}^{*} \simeq 4 \mathrm{MeV} /$ nucleon); $\mathrm{b}$ ) an intermediate source, emitting both light particles and IMF. In this second case, fragments are more neutron rich than the average matter of the overall system, and have a very different charge distribution, with respect to those statistically emitted from the QP. The above features can be considered as a signature of the dynamical origin of the midvelocity emission. The results of this analysis show that IMF can be produced via different mechanisms simultaneously present within the same collision. They also show that the probability of IMF production via dynamical reaction increase with the size of the target nucleus.
\end{abstract}

\section{Introduction}

The multifragmentation is one of the main de-excitation mode in the Fermi energy regime (30-50 MeV/nucleon). In this energy domain, for central collisions, this phenomenon has been successfully described by statistical approaches. Similarly, for midperipheral collisions, many experimental evidences have shown that the formation of a neck-like structure joining QT and QP can occur; this process leads to the production of IMF of dynamical origin.

The multifragmentation observed in central collisions, with the formation of a unique equilibrated emitting source, is in agreement with predictions of statistical models [1] suggesting that multifragmentation may result from a phase transition near the critical point. On the contrary, processes leading to the formation of neck-like structures can not be described on the basis of statistical approaches and are connected to the dynamics of the collision $[2,3,4]$. Therefore, one of the central issues concerning the multifragmentation process is the interplay between the attainment of the thermal and chemical equilibrium of the excited nuclear matter prior to the production of fragments and the dynamics of 
their emission.

We present and discuss data on ${ }^{58} \mathrm{Ni}+{ }^{58} \mathrm{Ni},{ }^{\text {nat }} \mathrm{Ag}$ midperipheral collisions at $30 \mathrm{MeV} /$ nucleon. We will compare the IMF production from different decay processes inside the same nuclear events. In fact, it will be investigated the role played by phenomena involving local, dynamically driven neck instabilities coupled to QP multifragmentation. A detailed comparison between the IMF produced via statistical and dynamical processes will be performed.

\section{Experimental method}

\subsection{Experimental set-up}

The experiment was performed at the INFN Laboratori Nazionali del Sud with the MEDEA [5] and MULTICS [6] experimental apparata. The angular range $3^{\circ}<\theta_{l a b}<28^{\circ}$ was covered by the MULTICS array [6]. The MULTICS array consists of 55 telescopes, each composed of an Ionization Chamber (IC), a Silicon position-sensitive detector (Si) and a CsI crystal. Typical energy resolutions were $2 \%, 1 \%$ and $5 \%$ for IC, Si and CsI, respectively. The identification thresholds in the MULTICS array were about 1.5 MeV/nucleon for charge identification. A good mass resolution for light isotopes (up to Carbon) was obtained. Energy thresholds for mass identification of 8.5, 10.5, 14 $\mathrm{MeV} /$ nucleon were achieved for ${ }^{4} \mathrm{He},{ }^{6} \mathrm{Li}$ and ${ }^{12} \mathrm{C}$ nuclei respectively. The $4 \pi$ detector MEDEA consists of a Barium Fluoride ball that covers polar angles from $30^{\circ}$ to $170^{\circ}$, and full azimuthal angle [5]. It is able to measure and identify light charged particles $(Z=1,2)$ and $\gamma$-rays up to about 300 $\mathrm{MeV}$.

\subsection{Data selection}

Standard methods, based on the accurate evaluation of the impact parameter [7], could not be used in this experiment, due to the relatively small number of detected fragments and particles $(\mathrm{Nc} \leq 5-6)$. Thus, the following approach was followed: 1) Collect only "complete" multifragment events, i.e. events where at least three IMF are produced and at least $80 \%$ of the total linear momentum are detected; 2) among them, define peripheral and midperipheral collisions when the heaviest fragment (with charge at least $1 / 3$ of that of the projectile, i.e. $Z \geq 9$ ) travels, in the laboratory frame, at a speed higher than $80 \%$ of that of the projectile $\left(\mathrm{v}_{P}=7.6\right.$ $\mathrm{cm} / \mathrm{ns}$ ). According to this, since the energy thresholds forbid the detection of QT reaction products, the total detected charge $\left(\mathrm{Z}_{T O T}\right)$ does not differ from that of the projectile by more than $30 \%\left(20 \leq \mathrm{Z}_{T O T} \leq 36\right)$.

\section{The midperipheral collisions}

The results presented hereafter will refer only to midperipheral events with at least three IMF, with the aim of observing the IMF emitting sources. In Fig. 1 the yields of carbon and oxygen fragments (for the $\mathrm{Ni}+\mathrm{Ni}$ case) are plotted as a function of the parallel component of the velocity, with respect to the beam axis. Two distinct regions can be observed, corresponding to an emission from a fast moving QP (with velocity around $6.5 \mathrm{~cm} / \mathrm{ns}$ ) and an IMF production at midvelocity $(3.8 \mathrm{~cm} / \mathrm{ns}$, around the center of mass velocity, due to the system symmetry). Similar results have been found in the $\mathrm{Ni}+\mathrm{Ag}$ data analysis.

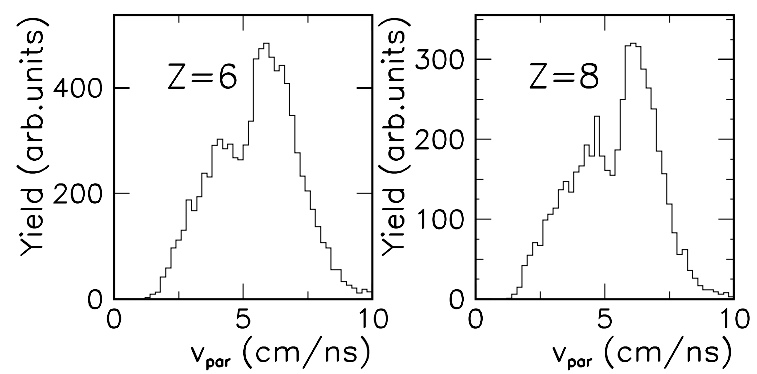

Figure 1. Parallel to beam component of the velocity for carbon and oxigen fragments.

These results show that in midperipheral collisions three different emitting sources are present; there are events in which the IMF can be simultaneously produced by the decay of QP and QT sources (QT fragments are not seen because below the energy threshold for identification), and from a neck, forming a midvelocity emission source. Thus the contributions from the QP and the neck sources must be disentangled in order to both improve the understanding of the IMF production mechanism and compare the IMF experimental yields with theoretical predictions.

To this purpose we study the process leading to the disassembly of the QP, and restrict the analysis to the QP fragments forward emitted (see Fig. 1). This constraint allows the selection of the decay products emitted in the QP decay with negligible contamination due to QT and midrapidity source emissions. We get indications that the QP reaches an equilibration stage before its de-excitation by studying, within its reference system, the angular (isotropic) and energy (maxwellian) distributions of the emitted fragments $[3,4]$. The results support the hypothesis that the QP has been subjected to an equilibration process, and it is therefore possible to compare the distributions with the predictions of the Statistical Multifragmentation Model (SMM) [1], that has already been succesfully tested [8] in the present excitation energy regime. The experimental charge distributions are properly reproduced by choosing excitation energies around $4 \mathrm{MeV} /$ nucleon.

Moreover, we extract the temperature $T$ through the method of double ratios of isotope yields [9]. The thermometers used suggest a break-up temperature of the QP decaying system of $3.9 \pm 0.2$ and $4.1 \pm 0.2 \mathrm{MeV}$ for the $\mathrm{Ni}+\mathrm{Ni}$ and $\mathrm{Ni}+\mathrm{Ag}$ reaction, respectively. Summarizing, the QP source has thermal characteristics $\left(E^{*} \simeq 4 \mathrm{MeV}, T \simeq 4\right.$ $\mathrm{MeV}$ ) for which multifragmentation takes place as the main de-excitation process.

Although the QP disassembly is governed by statistical models (once thermal equilibrium has been reached), the midvelocity emission exhibits quite different features that cannot be described in terms of statistical equilibrium. In 
particular, there are significant differences in the charge distribution and isotopic composition of the emitted fragments. For instance, the charge distribution of IMF emitted from the neck region has a higher yield between Carbon and Oxygen, when compared to the corresponding yield of fragments from $\mathrm{QP}$ (at fixed $\mathrm{Z}$ value). Indeed, the ratio of the two yields shows a classical bell-shape [2, 3], clear indication that the IMF fragments are the product of a dynamical process (see for instance Fig.4a of Ref.[3]).

Another aspect that distinguishes dynamical from statistical IMF is the neutron content. In particular those coming from the neck rupture are more neutron rich than the average matter of the systems, while in the case of the QP fragments the average values of the N/Z ratio for each element are similar to those of stable nuclear matter. In Fig. 2 the ratio between the two contributions as a function of the mass number for different $\mathrm{Z}$ values is presented (for the $\mathrm{Ni}+\mathrm{Ni}$ reaction).
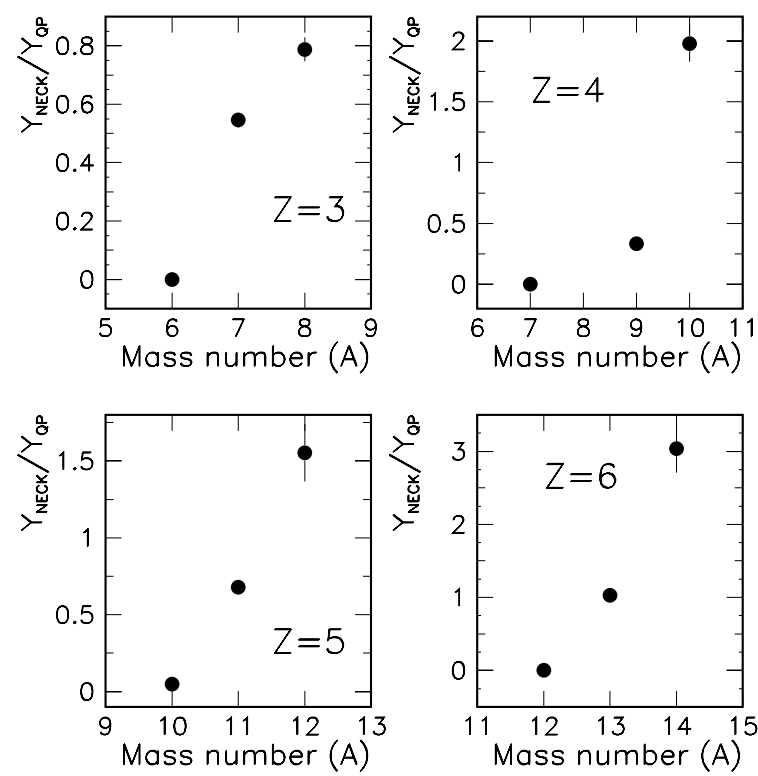

Figure 2. Yield ratios between the contribution of the midvelocity and of the QP as a function of the mass number.

\section{IMF dynamical production and the entrance channel of the reaction}

From the analysis of the two reactions $(\mathrm{Ni}+\mathrm{Ni}, \mathrm{Ag})$ it has been possible to select events where IMF are produced both via statistical and dynamical channels and to compare them. The QP (Ni-like nucleus) keeps its statistical characteristics $\left(E^{*} \simeq 4 \mathrm{MeV}, T \simeq 4 \mathrm{MeV}\right)$ in the two measurements. Instead, some differences appear in the amount of IMF coming from the neck rupture in the two reactions, in particular the $\mathrm{Ni}+\mathrm{Ag}$ midperipheral collisions show a copious production of dynamical IMF. In Fig. 3 the double ratio $\left(Y_{N e c k} / Y_{Q P}(N i+A g) / Y_{N e c k} / Y_{Q P}(N i+N i)\right)$ as a function of the $\mathrm{Z}$ value is presented; one can see that in the $\mathrm{Ni}+\mathrm{Ag}$ system we are in the presence of a production of dynamical IMF almost twice that of the lighter system
$(\mathrm{Ni}+\mathrm{Ni})$. This fact could be interpreted in terms of the larger size of the target that yields to an easier neck formation. Also, one must consider that in the $\mathrm{Ni}+\mathrm{Ag}$ case the $\mathrm{N} / \mathrm{Z}$ ratio is slightly higher than in the $\mathrm{Ni}+\mathrm{Ni}$ reaction $(1.21 \mathrm{Vs} 1.07)$ giving preferentially raise to the production of neutron rich nuclei (as those coming from the neck rupture).

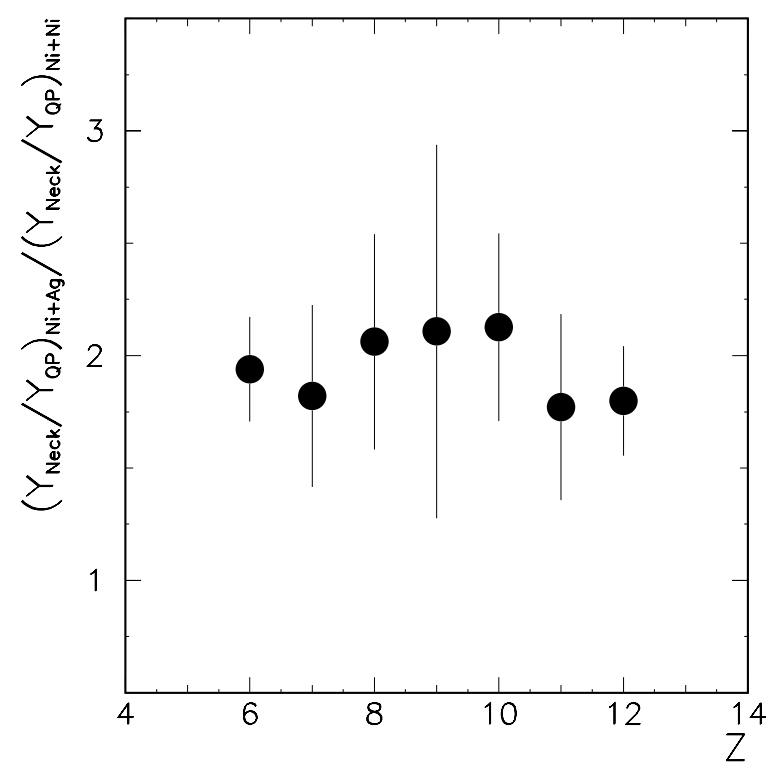

Figure 3. Double ratios $\left(Y_{N e c k} / Y_{Q P}(N i+A g) / Y_{N e c k} / Y_{Q P}(N i+\right.$ $\mathrm{Ni}$ )) between the contribution to IMF production of midvelocity and of QP emission, as a function of the atomic number.

\section{Conclusions}

The $\mathrm{Ni}+\mathrm{Ni}$,Ag $30 \mathrm{MeV} /$ nucleon experimental measurements were performed at the Superconducting Cyclotron of the INFN Laboratori Nazionali del Sud, Catania. By focusing the attention on multifragmentation events, it was finally possible to identify different sources of fragments in midperipheral collisions. The QP has been fully characterized and its decay follows the pattern of a statistical multifragmentation. The properties of the QP were investigated measuring its temperature and evaluating its excitation energy. It has been observed that IMF production is simultaneously present at midvelocity, due to dynamical processes that involve the overlap of projectile and target during the collision. These IMFs show a very different behaviour in terms of charge distribution and isotopic content of the fragments. Both features can be considered as a signature of a non statistical nature, and in fact the charge distribution can not be predicted by statistical models.

Finally, by comparing dynamical and statistical IMF characteristics, it appears that the first ones are more neutron rich than the average matter of the overall system, and that the difference of the two analyzed channels (namely the difference in target size) influences the dynamical production of IMF. 


\section{References}

[1] J.P. Bondorf et al., Nucl. Phys. A444, 460 (1986); A. S. Botvina et al., Nucl. Phys. A475, 663 (1987); J.P. Bondorf et al., Phys. Rep. 257, 133 (1995).

[2] C. P. Montoya et al., Phys. Rev. Lett., 73, 3070 (1994); J. Toke et al., Phys. Rev. Lett., 75, 2920 (1995); J. F. Dempsey et al., Phys. Rev. C 54, 1710 (1996); Y. Larochelle et al., Phys. Rev. C 55, 1869 (1997); J. Lukasik et al., Phys. Rev. C 55, 1906 (1997); P. Pawlowski et al., Phys. Rev. C 57, 1711 (1998); E. Plagnol et al., Phys. Rev. C 61, 014606 (1999); R.Wada et al., Phys. Rev. C 62, 034601 (2000); M.Veselsky et al., Phys. Rev. C 62, 041605(R) (2000); Y. Larochelle et al., Phys. Rev. C 62, 051602(R) (2000); J. Lukasik et al., Phys. Lett. B566, 76 (2003).

[3] P.M.Milazzo et al., Phys. Lett. B509, 204 (2001).

[4] P.M.Milazzo et al., Nucl. Phys. A703, 466 (2002).

[5] E. Migneco et al., Nucl. Instr. and Meth. Phys. Res. A314, 31 (1992).

[6] I. Iori et al., Nucl. Instr. and Meth. Phys. Res. A325, 458 (1993).

[7] C. Cavata et al., Phys. Rev. C 42, 1760 (1990).

[8] M. D’Agostino et al., Phys. Lett. B371, 175 (1996).

[9] S. Albergo et al., Nuovo Cimento 89, 1 (1985). 